\title{
Herstellung von Luftbildkarten in der Provinz Ta'izz (Arabische Republik Jemen)
}

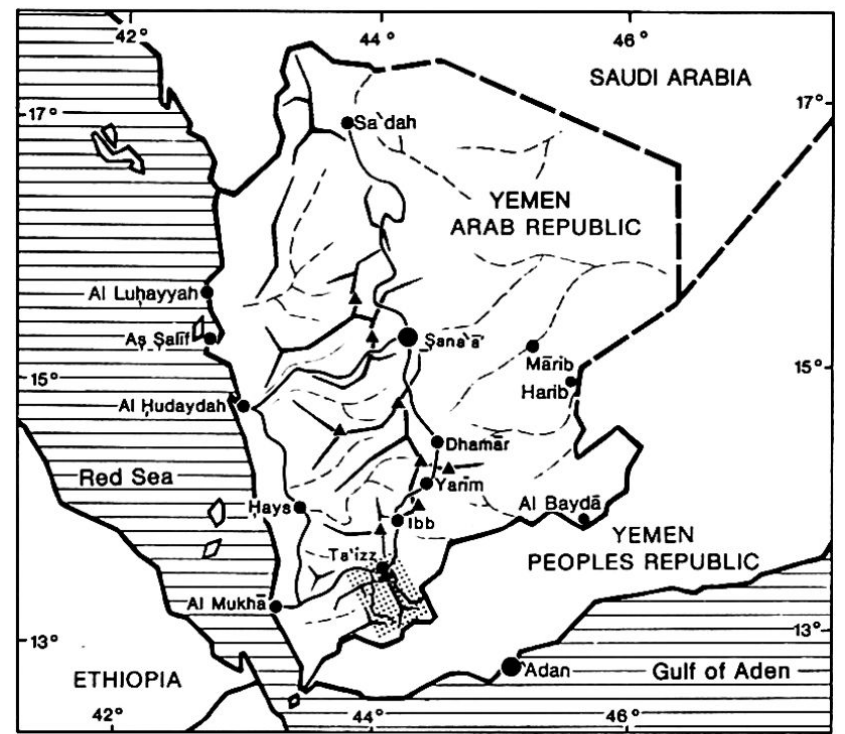

Fig. 1 Kartenskizze der Arabischen Republik Jemen (punktiert: Region Ta'izz-Turba-Hayfan)

Dank dem Interesse der jemenitischen Regierung und der Unterstützung durch UNICEF (United Nations' Children Fund), FAO (Food and Agriculture Organisation) und DrTZ (Dienst für Technische Zusammenarbeit, Bern) konnten die im vorliegenden Beitrag beschriebenen vier Luftbildkarten für die Provinz Ta'izz (Arabische Republik Jemen) hergestellt werden.

Die Blätter «Ta'izz», «Turbah», «Wadi al Barakani» und «Hayfan» (Maßstab 1:25000) können am Geographischen Institut der Universität Zürich bezogen werden.

\section{Summary}

This article deals with a simple method to produce photomaps of as yet unsurveyed areas. Since a triangulation network or a topographic inventory of the areas to be mapped did not exist, it was considered most suitable to produce some kind of «preliminary maps»: Maps of medium accuracy but high content of information which could be produced within a short time with modest technical means and at low cost. The following procedure was found to be most suitable for that purpose.

A network of control points is established by means of the slotted templet method. Aerial photographs, dodged by the Log Etronic procedure, are assembled to semicontrolled mosaics. Grestlines, plateau edges and symbols for mountain peaks, passes etc. as well as spot heights, roads, tracks, wells, market places and names of settlements, districts and surface features are introduced into the photomosaics by means of transparent overlays. This latter information was collected during a three months' field survey in the Yemen Arab Republic.

\section{Einleitung}

Der vielseitige Gebrauch von Karten ist in den entwickelten Ländern zur Selbstverständlichkeit geworden. Eine reichhaltige Auswahl von Plänen, topographischen und thematischen Karten steht uns zur Verfügung.

In manchen Entwicklungsländern hingegen darf noch nicht mit dem Vorhandensein von zweckdienlichen Karten und Plänen gerechnet werden.1) In Gebieten, wo weder regionale Übersichten noch zuverlässige statistische Angaben bestehen, stellen Luftbild und Satellitenaufnahme besonders wertvolle, zeit- und kostensparende Hilfsmittel zur Grundlagenbeschaffung dar. Die Entwicklung und Prüfung ihrer Anwendungsmöglichkeiten in wenig erforschten Gebieten ist deshalb von besonderer Bedeutung. Für Geographen und Luftbildinterpreten stehen dabei folgende Problemstellungen im Vordergrund:

1) Entwicklung zweckmäßiger Verfahren zur Herstellung von kartographischen Unterlagen:

- zur Übersicht in wenig bekannten Gebieten,

- zur Orientierung im Gelände,

- als Kartierungsgrundlage bei Felduntersuchungen,

- als Planungs- und Projektunterlagen.

2) Entwicklung von Methoden zur direkten Erhebung und Darstellung von Daten aus Luftbild und Satellitenaufnahme:2)

- zur Geologie, Hydrographie, Pedologie,

- zur Landnutzung (Agrarstatistik, Ertragsschätzungen),

- zur Demographie (Haus-, Bevölkerungsverteilung) u. a. m.

Hans Steffen, dipl. geogr., Geographisches Institut der Universität, Blümlisalpstraße 10, 8006 Zürich. 
Der nachfolgende Beitrag beschränkt sich auf den erstgenannten Problemkreis. Es wird versucht, einen Überblick über Ausgangssituation, Zielsetzung und angewandte Methoden bei der Kartenherstellung in der Arabischen Republik Jemen zu vermitteln.

\section{Ausgangsituation}

In den vergangenen Jahrhunderten galt der Jemen als ein schwer zugängliches Land. Obgleich von 1538 bis 1635 und 1848-1918 nominal zum Osmanischen Reich gehörend, blieb er von technischen Neuerungen und äußeren Entwicklungen weitgehend unberührt. ${ }^{3)}$ Auch nachdem das Land sich anfangs dieses Jahrhunderts aus dem Osmanischen Reich gelöst hatte, verblieb es in traditioneller Isolation; nur wenige Forscher erhielten die Erlaubnis, den Jemen zu besuchen.4)

Nach der Absetzung des letzten Imams, Muhammad al Badr, und der Proklamation der Republik 1962, kam es im Jemen zu einem erbittert geführten Bürgerkrieg zwischen Republikanern und imamtreuen Royalisten.5) Die Auseinandersetzungen konnten 1969/70 beigelegt werden. Somit steht der Jemen erst seit wenigen Jahren für technische Aufbauarbeiten offen.6)

Bis heute wurden im Jemen noch keine Arbeiten zur Landesvermessung und nur wenige topographische Geländeaufnahmen durchgeführt. Mit wenigen Ausnahmen wurden die zur Zeit erhältlichen Übersichtskarten ohne genügende Feldarbeit hergestellt. Darum sind Fehler häufig, vorab im Namenmaterial, bei Verkehrswegen, Siedlungen und Höhenangaben.

Das Fehlen von mittel- und großmaßstäbigen Karten erschwert die Planung und Durchführung zahlreicher Projekte. Deshalb wurden 1970/71 für einige Projekte des United Nations' Development Program (UNDP) Luftbilder hergestellt, darunter auch für die Region Ta'izz-Turbah-Hayfan. ${ }^{7)}$ Obwohl diese Luftbilder in den noch unkartierten, kaum bekannten Gebieten einzigartige, wertvolle Dokumente darstellen, werden sie für Arbeiten im Felde doch relativ selten gebraucht. Folgende Nachteile des Luftbildes sind im allgemeinen bei Arbeiten im Gelände zu beachten:

1) Luftbilder sind relativ teuer, empfindlich und werden leider oft als Geheimdokumente betrachtet; die zuständigen Stellen leihen sie darum nur selten und ungern aus.
2) Ausgehend von einer größeren Anzahl von Luftbildern (Format max. $23 \times 23 \mathrm{~cm}$ ) ist es nicht leicht, eine Gebietsübersicht zu gewinnen oder großräumige Gebietsvergleiche anzustellen.

3) Die detaillierte Interpretation und Auswertung des reichen Photoinhaltes ist kaum ohne Fachkenntnisse, Interpretationsschlüssel usw. durchzuführen.

4) Da Luftbilder keine Namen, Höhenangaben, StraBenklassifikation und dgl. enthalten, eignen sie sich nur in beschränktem Maße zur Orientierung im Gelände.

\section{Problemstellung und Zielsetzung}

Es stellt sich somit die Frage, wie in noch unvermessenen, unkartierten Gebieten aus Luftbildern nach einfachen Methoden, mit bescheidenem finanziellem und zeitlichem Aufwand nützliche Karten hergestellt werden können.

Zwar sind die Methoden der Herstellung von Linienkarten, basierend auf Aerotriangulation und photogrammetrischer Auswertung entwickelt und bekannt.8) Diese in industrialisierten Ländern normalerweise angewandten Verfahren setzen jedoch zahlreiche Grundlagen wie Landvermessung, Signalisierung vor dem Bildflug, terrestrische Vermessung von Paßpunkten, terrestrische Bestandesaufnahmen voraus; Grundlagen, die in manchen Entwicklungsländern noch nicht vorhanden sind. Vielerorts muß zur Bewältigung des Notwendigsten auf «europäische» Perfektion und Genauigkeitsnormen verzichtet werden. Den dringendsten Bedürfnissen und Aufgaben vermögen häufig schon «preliminary maps» (prov. Ausgaben) zu genügen; zu dieser Kategorie zählen beispielsweise auf Luftbildmosaiken beruhende Karten mittlerer Genauigkeit, jedoch mit reichem thematischem Photoinhalt.

Mit Rücksicht auf die im Jemen herrschende Ausgangssituation wurde folgende Zielsetzung für die Luftbildkarten der Region Ta'izz-Turbah-Hayfan gewählt:

1) Kartographische Darstellung eines möglichst groBen, bis dahin unvermessenen und wenig bekannten Gebietes $\left(4 \times 300 \mathrm{~km}^{2}\right)$;

2) Bescheidener finanzieller Aufwand: $5-10 \%$ der Herstellungskosten von einfachen topographischen Karten 1:25000; 


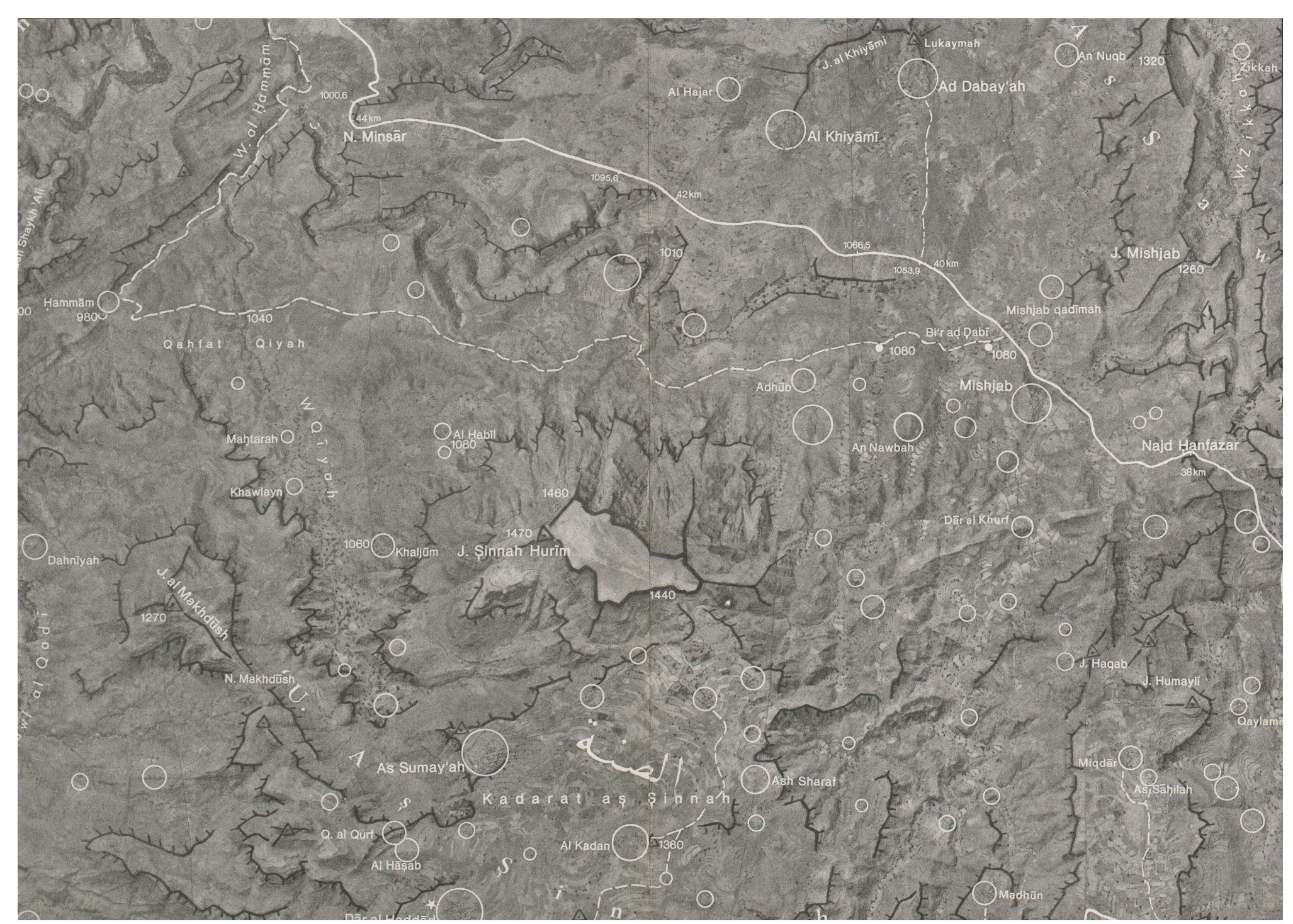


3) Herstellung im 1-Mannbetrieb, ohne SpezialistenTeam; daher Einsatz moderner, aber technisch einfacher Verfahren;

4) Kurze Herstellungszeit: Montage, kartographische Bearbeitung und Reproduktion in 2-3 Monaten pro Kartenblatt;

5) Einhalten einer mittleren Genauigkeit: Distanzmessungen innerhalb $\pm 10 \%$, Höhenangaben $\pm 20 \mathrm{~m}$;

6) Mehrzweckkarte: optimale Ausnützung des hohen thematischen Informationsgehaltes der Luftbilder.

\section{Herstellungsverfahren}

Ausgehend von der erwähnten Zielsetzung wurden zahlreiche einfache Verfahren zur Herstellung von Luftbildkarten überprüft. Die schließlich ausgewählten und angewandten Methoden seien nachfolgend kurz beschrieben:9)

Vom Original-Luftbildfilm werden Kontaktabzüge mit dem LOG ETRONIC-Kontrastausgleichsverfahren hergestellt; dieses Kopiersystem behebt auffällige Gegenlicht/Mitlichtgegensätze und Helligkeitsunterschiede, die auf dem randlichen Lichtabfall des Objektivs beruhen. Damit ermöglicht dieses Verfahren die Herstellung von Luftbildausschnitten, die in Grautonumfang und Kontrastverhältnissen weitgehend übereinstimmen (Abb. 2 und 3).

Die Kontaktkopien werden nach einem vorgegebenen Netz von Paßpunkten (x/y-Koordinaten) zu semikontrollierten Luftbildmosaiken zusammengesetzt. Das notwendige Netz von Paßpunkten kann mit Hilfe der Radialschlitz-Triangulation (Slotted Templet Methode) gewonnen werden. Für dieses Verfahren sind ein flächenhafter Bildflug (mehrere Flugstreifen mit etwa $20 \%$ Quer- und 60\% Längsüberdeckung) und nur geringe terrestrische Vermessungsgrundlagen (z. B. drei astronomisch eingemessene Fixpunkte) erforderlich. Infolge der Radialdeformation können in semikontrollierten Luftbildmosaiken entlang den Schnittlinien benachbarter Luftbildausschnitte Doppelabbildungen, Bildauslassungen oder -versetzungen auftreten. Um Fehldeutungen beim Kartenlesen zu ver-

Beilage:

Originalausschnitt aus Luftbildkarte 1 : 25 000, im Lichtdruckverfahren reproduziert. meiden, und um die markantesten Relieferscheinungen deutlicher hervorzuheben, wird eine erste kartographische Bearbeitung (Abb. 4) vorgenommen:

Eintrag von Bergkämmen, -gipfeln, Paßübergängen, Terrassenkanten usw. Die Geländezeichnung wird mit einer Maske schwarz ins Luftbildmosaik eingeblendet (Abb. 5).

Von den maskierten Mosaikvorlagen werden durch photographische Vergrößerung Halbtonnegative im Maßstab 1:25000, Format $70 \times 90 \mathrm{~cm}$ hergestellt.

Eine topographische Bestandesaufnahme im Felde bildet die Grundlage für die zweite kartographische Bearbeitung der Luftbildmosaike (Abb. 6). Mit Hilfe eines transparenten Deckers auf den Halbtonnegativen können Namen, Straßen, Höhenkoten, Signaturen für Siedlungen, Schulen, Brunnen, Quellen usw. weiß in die Mosaike eingeblendet werden (Abb.7). Die Reproduktion der Luftbildkarten erfolgt im Lichtdruck, einem qualitativ hochstehenden Druckverfahren, das Halbtöne ohne Aufrasterung des Bildes wiederzugeben ermöglicht (Abb. 8).

\section{Feldarbeit}

Die Feldarbeit diente dazu, Informationen, die den Luftbildern nicht entnommen werden konnten, zu sammeln. Sie konzentrierte sich daher vor allem auf:

- Erhebung von Namen der Siedlungen, Berge, Paßübergänge, Wadis usw.

- Erfassen der öffentlichen Dienste: Schulen, Märkte, Spitäler;

- Abgrenzen der Verwaltungsregionen im Gelände;

- Eintragen und Klassieren bestehender Verkehrswege;

- Bestimmen von Höhenkoten;

- Erarbeiten eines Interpretationsschlüssels zur Landnutzung und Geologie.

Als wichtigstes Hilfsmittel für die Felderhebungen standen provisorische Mosaikkopien zur Verfügung (Abb. 5). Mit deren Hilfe konnten Marschrouten und -etappen zum voraus geplant, die Orientierung im Gelände erleichtert und die gesammelten Informationen ortsbezogen festgehalten werden.

Aus den handgeschriebenen Steuerregistern, die in den Distrikthauptorten aufbewahrt werden, konnten die wichtigsten Dorfnamen der zu bearbeitenden Ge- 
Fig. 2-7 Die Arbeitsvorgänge zur Herstellung der Luftbildkarten. Ausschnitt aus Blatt «Wadi al Barakani», 4fache Verkleinerung.

Fig. 2 Luftbildmosaik, ohne Kontrastausgleich hergestellt.

Fig. 4 Inhalt von Maske I. Eintragungen zum Relief.

Fig. 6 Inhalt von Maske II. Topographische Ergänzungen.
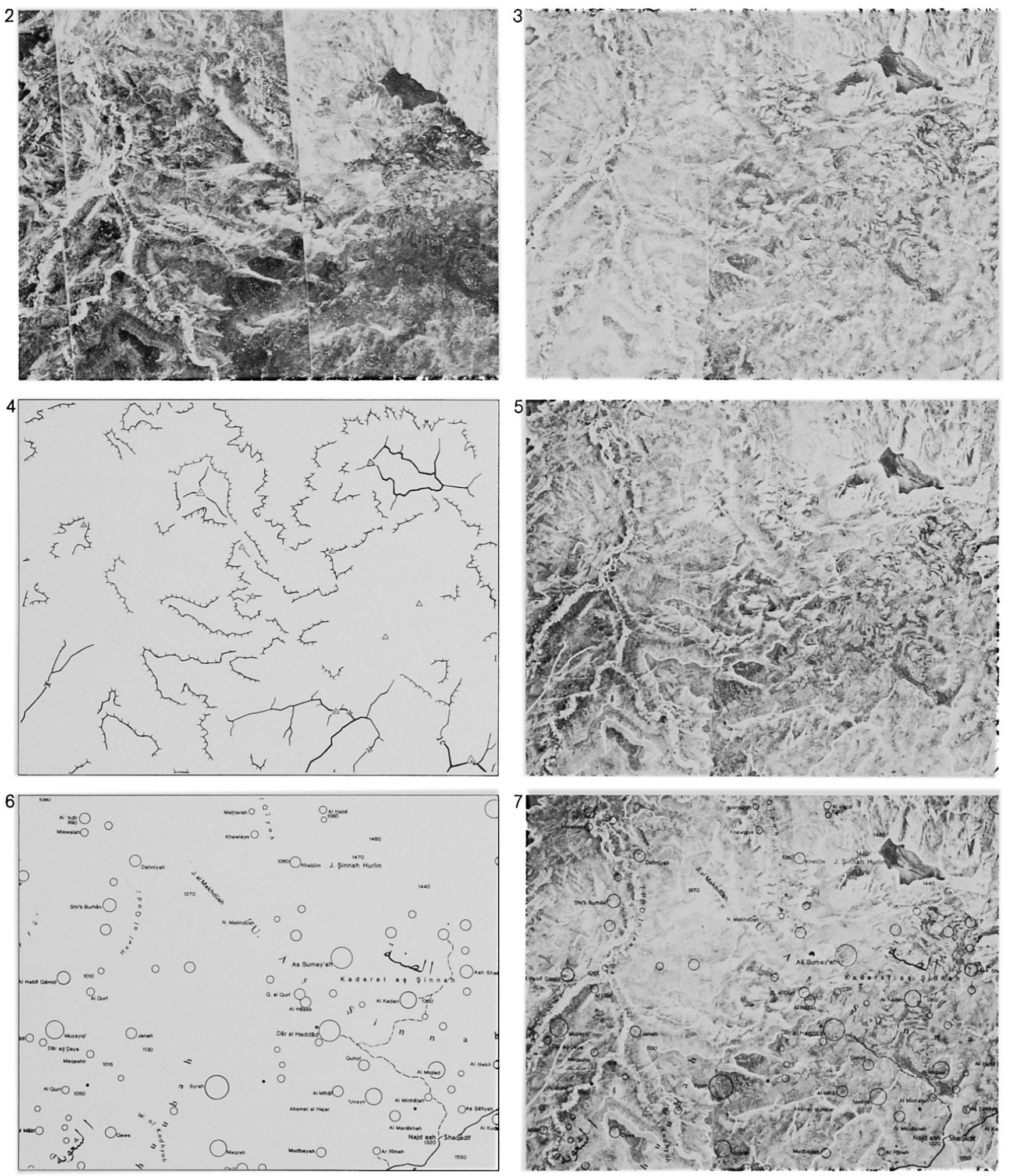

Fig. 3 Luftbildmosaik, im Log Etronic-Kontrastausgleichsverfahren hergestellt.

Fig. 5 Kontrastausgeglichenes Luftbildmosaik mit Eintragungen zum Relief.

Fig. 7 Endgültige Luftbildkarte mit Eintragungen zum Relief und topographischen Ergänzungen. 
biete abgeschrieben werden. Da aber auf den jemenitischen Amtsstellen weder kartographische Unterlagen, noch irgendwelche Angaben über die Lage dieser Ortsnamen verfügbar waren, konnte das Namenmaterial nur aufgrund von Feldbegehungen in die Karten eingetragen werden. An Ort und Stelle mußte die einheimische Bevölkerung nach den Namen ihres Dorfes, der umgebenden Berge, Täler, Pässe oder nach der Lage des nächsten Brunnens, Schulhauses, Wochenmarktes usw. befragt werden.

Die Qualität der mündlichen Auskünfte fiel jedoch sehr unterschiedlich aus, teils aus Unverständnis, teils aus Mißtrauen. Anstelle des Dorfnamens wurde beispielsweise häufig der Distrikt- oder Provinzname erwähnt; was sollte denn einen Fremden der Name eines kleinen, abgelegenen Weilers interessieren! Hin und wieder traten auch Meinungsverschiedenheiten bei der Namengebung auf. Deshalb kam der Bewertung der Aussagen nach ihrer Vertrauenswürdigkeit besondere Bedeutung zu; eine äußerst schwierige Aufgabe für einen Fremden mit geringen Sprach- und Personenkenntnissen. Ein aus der Gegend stammender, sehr zuverlässiger Mitarbeiter (Qadi Muhammad Abdullah Salih) war in dieser Hinsicht äußerst hilfreich als ständiger Begleiter, Schreiber, Träger und Koch.

Das schriftlich und auf Tonband festgehaltene arabische Namengut (total ca. 1300 Namen) wurde nach dem «BCG-PCGN 1956 System for Arabic» in die lateinische Schrift übertragen.

\section{Schlußbetrachtung}

In verschiedener Hinsicht stellen die nun vorliegenden Luftbildkarten eine Kompromißlösung dar. Einerseits mußte auf hohe photogrammetrische Genauigkeit bewußt verzichtet werden; zudem waren gewisse Fehler bei der erstmaligen Aufnahme und Lokalisierung von Ortsnamen kaum zu vermeiden. Andererseits konnten Zeitaufwand und Herstellungskosten sehr niedrig gehalten werden, da relativ wenige zeichnerische und kartographische Arbeiten anfielen. Dank dem reichen Inhalt der Photographie geben die Luftbildkarten über zahlreiche, geographisch interessante Erscheinungen Aufschluß, deren zeichnerische Wiedergabe einen unverhältnismäßig größeren Aufwand erfordert hätte. Es sei nur auf ein paar Beispiele hingewiesen:
Zur Geologie: Die Gliederung in präkambrisches Grundgebirge, mesozoische Formationen (Horste und Hochplateaus bildend) und tertiäre Trappablagerungen (Gebirge mit deutlicher, rel. dunkler Schichtung) ist in den Karten gut erkennbar (siehe Blatt "Turbah»). Verschiedene Gangsysteme mit Basaltfüllungen treten als Härtlinge prächtig zutage (siehe Blatt «Barkani»).

Zur Landnutzung: Die oft extrem dichte Terrassierung vermittelt ein eindruckvolles Bild des intensiven Ackerbaus bis hinauf an die steilsten Gebirgshänge. Da die Luftbilder in der Trockenzeit (Febr. 1971) aufgenommen wurden, erscheinen nur die regelmäßig bewässerten Flußoasen, sowie Kaffee- und Qatanbaugebiete dunkel, die brachliegenden Felder dagegen hell.

Zur Demographie: Im Maßstab 1:25000 können die Häuser größtenteils noch einzeln im Luftbild erkannt werden; Untersuchungen über verschiedene Haustypen, Siedlungsmuster, bevorzugte Wohnlagen usw. lassen sich anhand der Karten anstellen.

Zahlreiche Verwendungsarten beweisen, daß die Karten für verschiedene Aufgaben vollauf genügen und zur besseren Kenntnis und Entwicklung der dargestellten Gebiete beitragen können. Der jemenitischen Regierung vermitteln sie erstmals eine Übersicht über die Bevölkerungsverteilung, Ausdehnung und Lage der überlieferten Verwaltungseinheiten, der landwirtschaftlichen Anbaugebiete, über vorhandene Verkehrswege usw. Verschiedenen Entwicklungsorganisationen dienen die Karten zur Orientierung im Gelände (z. B. bei der Durchführung von Impfprogrammen in ländlichen Gebieten), zur Planung landwirtschaftlicher Projekte und als Kartierungsgrundlage für geologische und pedologische Aufnahmen.

1774 setzte Carsten Niebuhr ${ }^{10}$ ) den folgenden Begleittext zur Veröffentlichung seiner Übersichtskarte des Jemens; die Anmerkungen passen auch für die nun vorliegenden Luftbildkarten:

«Diese Karte des Jemens ist zwar nicht so genau und vollständig, als andere, die in Europa von ganzen Gesellschaften gemessen und gezeichnet worden sind. Ich glaube aber doch, daß diejenigen, welche wissen wie schwer und gefährlich es ist, in den Gebieten eines fremden Herrn Messungen anzustellen und geographische Nachrichten zu sammeln, damit zufrieden sein werden und daß sogar von manchen Gegenden in Europa noch keine genaueren Karten bekannt sind.» 


\section{Literaturauswahl}

1) HAGEN, T.: Ein neuer Weg der schweiz. Entwicklungshilfe? NZZ, Nr. 417, 8. Sept. 1971

2) CALLEGARI, R./STEFFEN, H.: Estimation of population and land-use data from aerial photographs in the Ta'izz-Turbah region, Geographisches Institut, Universität Zürich 1974

3) PAWELKE, G.: Der Jemen, das verbotene Land, Düsseldorf 1959

4) RATHJENS, C. und VON WISSMANN, H.: Rathjens-von Wissmansche Südarabienreise, Band III, Abh. aus dem Gebiet der Auslandkunde, Hamburg 1934

5) SCHMIDT, D. A.: Yemen - the Unknown War, London 1968

6) FAYEIN, C.: Yemen, Paris 1975

7) UNDP Information Paper, YAR, No. 9, compiled report on the UNDP/FAO projects
8) VAN ZUYLEN, L.: Some remarks about the development of the application and reproduction of photomaps during the last four years, ICA Conference, Canada 1962

SCHWEISSTHAL, R.: Grundlagen, Bearbeitung und Herstellung großmaßstäbiger Luftbildkarten, Hannover 1969

SCHMIDT-FALKENBERG, H.: Topographische Karte 1:25000 (Luftbildkarte), in Bildmessung und Luftbildwesen 3/74, S. 74-80

9) STEFFEN, H.: Herstellung von Luftbildkarten in unvermessenen Gebieten - ein Beitrag zur angewandten Geographie in Entwicklungsländern, Diplomarbeit, Geographisches Institut Universität Zürich, Zürich 1974

10) NIEBUHR, C.: Beschreibung von Arabien und anderen umliegenden Ländern, 1. Band, Amsterdam 1774 